\title{
Microsurgical Treatment of Sporadic and von Hippel-Lindau Disease Associated Spinal Hemangioblastomas: A Single-Institution Experience
}

\author{
Joe M. Das, Krishnakumar Kesavapisharady, Saravanan Sadasivam, Suresh Narayanan Nair \\ Department of Neurosurgery, Sree Chitra Tirunal Institute for Medical Sciences and Technology, Thiruvananthapuram, India
}

\section{Study Design: Retrospective cohort study.}

Purpose: To examine the clinical profile and surgical complications in patients with spinal hemangioblastomas and to evaluate the long-term outcome in them.

Overview of Literature: Although considered to be histologically benign, hemangioblastomas may cause significant neurological deficits. The proportion of spinal hemangioblastomas associated with von Hippel-Lindau (VHL) disease has been estimated be $13 \%-59 \%$. Preoperative neurological function correlates with postoperative neurological status. Studies have shown no difference in outcomes between sporadic and VHL-associated spinal hemangioblastomas.

Methods: This retrospective study included 14 consecutive patients treated for spinal hemangioblastomas at our institute between January 2000 and June 2013. The mean follow-up period was 5 years. Magnetic resonance imaging of the complete neuraxis was performed in all cases, and preoperative embolization was performed in two cases.

Results: In total, 14 patients underwent 18 surgeries, of which 15 were for spinal hemangioblastomas. Of all the patients, 86\% had motor weakness and $79 \%$ presented with sensory disturbances. Preoperative McCormick functional grades were grade I in 7 $(50 \%)$, grade II in $3(21 \%)$, and grade III in $4(29 \%)$ patients; $50 \%$ patients were diagnosed with VHL disease. All patients underwent complete resection of the tumor. Eight patients experienced deterioration in their neurological status in the immediate postoperative period; among them, five had gradual improvement. At 5-year follow-up, 11 (78.57\%) patients showed good functional outcomes.

Conclusions: Microsurgical excision of spinal hemangioblastomas can cause postoperative morbidity, mainly in the form of neurological deterioration. Almost half of our patients had deterioration in the McCormick grade in the immediate postoperative period. However, a complete microsurgical excision can result in good long-term functional outcomes, as most of the immediate postoperative neurological deterioration in our patients was reversible. There was no difference in the long-term functional outcomes between sporadic and VHL-associated spinal hemangioblastomas.

Keywords: Spinal cord; Spinal cord neoplasms; Hemangioblastoma; von Hippel-Lindau disease; Microsurgery

\section{Introduction}

Hemangioblastomas are benign tumors of the central ner- vous system (CNS), which can present as sporadic lesions (75\%) or as a part of a multisystem involvement in von Hippel-Lindau (VHL) disease (25\%). The manifestations

\footnotetext{
Received Oct 10, 2016; Revised Dec 28, 2016; Accepted Feb 3, 2017

Corresponding author: Joe M. Das

Department of Neurosurgery, Sree Chitra Tirunal Institute for Medical Sciences and Technology,

Medical College P.O., Thiruvananthapuram, Kerala, India. PIN-695 011

Tel: +91-9447092432, Fax: +91-4712446433, E-mail: drjoemdas@yahoo.co.in
} 
of VHL syndrome are renal and pancreatic cysts, renal cell carcinoma (RCC), pheochromocytoma, endolymphatic sac tumors, pancreatic islet tumors, and epididymal and broad ligament cystadenomas [1].

VHL syndrome is associated with a germline mutation of the VHL tumor suppressor gene in the short arm of chromosome 3 (3p25.3) and is inherited as a highly penetrant autosomal dominant trait [2]. De novo mutations of the VHL gene are also demonstrated in sporadic diseases. The spinal cord is a frequent site of hemangioblastomas in patients with VHL syndrome, accounting for more than $40 \%$ of VHL-associated CNS lesions detected on magnetic resonance imaging (MRI) [3]. Spinal hemangioblastomas cause significant morbidity to the patients because of frequent neurological deficits, often secondary to edema, cyst, and syrinx. Fortunately, the morbidity and mortality caused by CNS hemangioblastomas have been reduced due to advances in imaging modalities, microsurgical techniques, screening, and early diagnosis of associated tumors.

We studied patients with both sporadic and VHLassociated spinal hemangioblastomas to analyze the presentation, management, course, and surgical outcomes of spinal hemangioblastomas.

\section{Materials and Methods}

\section{Patient population}

This retrospective study included 14 patients who underwent surgery for spinal hemangioblastoma at our department from January 2000 to June 2013. Case records and operative reports were retrospectively examined after obtaining approval from the Institutional Ethics Committee number (SCT/IEC/665/August 2014). All patients had undergone preoperative MRI of the whole spine. At our institute, the investigations performed in patients with spinal hemangioblastoma to rule out the associated VHL syndrome include the following: MRI of the brain (to rule out intracranial hemangioblastomas); ultrasound of the abdomen, pelvis, and scrotum (to rule out visceral cysts or other lesions); computed tomography of the abdomen in selected cases (if ultrasound is showing doubtful lesions); and fundoscopy (to rule out retinal hemangioblastoma).

The neurological function of the patients was graded according to the clinical scale described by McCormick et al. (Table 1). Functional grade was assessed in the preoperative and immediate postoperative periods as well as at 1- and 5-year follow-up. Functional outcomes were compared between the sporadic and VHL-associated cases.

\section{Statistical method}

Mann-Whitney U test was used to collate the outcomes between the sporadic and VHL-associated spinal hemangioblastomas.

\section{Results}

In total, 14 patients $(\mathrm{n}=14 ; 8$ [57\%] females and 6 [43\%] males with mean age 33.7 years; age range, $16-55$ years) underwent 18 surgeries, of which 15 were for spinal hemangioblastomas and 3 for cerebellar hemangioblastomas. One patient underwent surgery twice for cerebellar hemangioblastomas on both sides, and one patient underwent surgery for right cerebellar hemangioblastoma. Furthermore, one patient underwent a simultaneous resection of cerebellar and cervical hemangioblastomas.

\section{Clinical presentation}

The duration of illness ranged from 2 weeks to 4 years, with the average duration being 6 months (median). Twelve patients (86\%) had motor weakness in the form of hemiparesis, quadriparesis, or paraparesis at presentation. Eleven patients (79\%) presented with sensory disturbances. Pain was the presenting symptom in 4 patients (29\%). Bladder disturbance was present in 3 patients (21\%). McCormick functional grade was assessed preoperatively,

Table 1. McCormick functional status scale

Grade I, Neurologically intact, may have minimal dyesthesia, normal gait

Grade II, Mild motor or sensory deficit, patient maintains functional independence

Grade III, Moderate deficit, limitation of function, independent with external aid

Grade IV, Severe motor or sensory deficit, limited function, dependent

Grade V, Paraplegia or quadriplegia, even with flickering movement 
at discharge, and at follow-up. Preoperative McCormick functional grades were grade I in 7 (50\%), grade II in 3 (21\%), and grade III in 4 (29\%) patients.

\section{Associations}

One (7\%) patient had a history of cerebellar hemangioblastoma in siblings. Three (21\%) patients had cerebellar hemangioblastomas associated with a spinal cord tumor. Five (36\%) patients had associated renal or pancreatic cysts. Two (14\%) patients had retinal disease. Based on the clinical criteria, 7 (50\%) patients were diagnosed with VHL disease. Of those seven, one had a positive family history and the others had multiple CNS hemangioblastomas. The mean age of patients with VHL disease was 32.5 years.

\section{Tumor location}

Of 14 patients, 8 (57\%) had the tumor in the cervical cord, $5(36 \%)$ in the thoracic spinal cord, and 1 in the conus (7\%). In all the patients, the tumor was dorsally located. Syrinx was present in 9 (64\%) patients.

\section{Therapeutic adjuncts}

Preoperative digital subtraction angiography (DSA) was performed in 11 (79\%) patients. Preoperative embolization was performed in two patients, of whom one had a tumor in the conus and the other in the dorsal cord.

\section{Surgical intervention}

All patients underwent posterior spinal laminectomy via posterior midline approach and a complete resection of the tumor.

\section{Postoperative course}

Eight $(57.1 \%)$ patients experienced deterioration in the neurological status in the immediate postoperative period; among them, three had tumors in the cervical cord and two in the dorsal cord. Of the eight patients, one had a recurrent tumor. Two patients had preoperative functional grade III, another two had grade II, and four had functional grade I. In one patient, neurological deterioration was transient and was functionally grade I at discharge.
None of the patients had wound infection in the postoperative period. Only one patient had cerebrospinal fluid (CSF) leak from the wound in the postoperative period. It was managed by re-suturing the wound.

\section{Follow-up}

The mean follow-up period was 5 years, ranging from 6 months to 8 years. Out of the eight patients who had postoperative neurological deterioration, six patients showed improvement in their neurological status-one of them became functionally grade I at the time of discharge; four became grade I and one became grade II at the end of 1-year follow-up. Other two patients had some improvement in their lower limb power, but they remained functionally dependent (McCormick grade III) at 5-year follow-up. Both had an improvement in their bladder function and no longer needed an indwelling urinary catheter.

\section{Tumor recurrence}

Tumor recurrence was noticed at the operative site in one (7\%) patient (a female) at 3-year follow-up. She also developed a new lesion at a site different from the operative site and pancreatic cysts. She underwent re-exploration excision of the recurrent tumor. Total tumor excision was achieved, but she developed paraplegia in the postoperative period (preoperative functional grade II). At 6-year follow-up, she had improvement in bladder function and was McCormick grade III.

\section{Surgical outcome}

There was $1(7 \%)$ mortality in the postoperative period. This patient (a male) developed intraventricular bleeding due to malignant hypertension in the postoperative period. His preoperative work-up for pheochromocytoma had been negative.

At 1-year follow-up, 8 (57.1\%) patients had good functional outcomes. Although a few patients developed neurological deterioration in the postoperative period, it was transient, and the neurological status of many of them improved at 1-year follow-up. At 5-year follow-up, 11 (78.57\%) patients had good functional outcomes.

Mann-Whitney U test was used to compare the outcomes between sporadic disease and VHL-associated 
Table 2. Table comparing features of sporadic $(n=7)$ and VHL-associated spinal hemangioblastomas $(n=7)$

\begin{tabular}{|c|c|c|c|}
\hline SI. no. & Feature & $\begin{array}{c}\text { Sporadic spinal } \\
\text { hemangioblastoma }\end{array}$ & $\begin{array}{l}\text { VHL-associated spinal } \\
\text { hemangioblastoma }\end{array}$ \\
\hline 1. & Age (yr, average) & 34.9 & 32.5 \\
\hline 2. & Sex (male:female) & $3: 4$ & $3: 4$ \\
\hline 3. & $\begin{array}{l}\text { Clinical findings (no. of patients): } \\
\text { 1. Paraparesis } \\
\text { 2. Hemiparesis } \\
\text { 3. Quadriparesis } \\
\text { 4. Hand muscle atrophy } \\
\text { 5. Bladder involvement } \\
\text { 6. No motor deficit }\end{array}$ & $\begin{array}{l}0 \\
0 \\
3 \\
2 \\
1 \\
2\end{array}$ & $\begin{array}{l}4 \\
3 \\
0 \\
0 \\
2 \\
0\end{array}$ \\
\hline 4. & Duration of symptoms (median in months) & 6 & 5 \\
\hline 5. & $\begin{array}{l}\text { Level(s) of the lesion: } \\
\text { 1. Cervico-medullary junction } \\
\text { 2. Cervical } \\
\text { 3. Thoracic } \\
\text { 4. Lumbar }\end{array}$ & $\begin{array}{l}1 \\
5 \\
3 \\
0\end{array}$ & $\begin{array}{l}1 \\
3 \\
4 \\
1\end{array}$ \\
\hline 6. & Average no. of lesions/patient & 1.42 & 1.42 \\
\hline 7. & Location in relation to cord (\%) & Dorsal (100) & Dorsal (100) \\
\hline 8. & Presence of syrinx or cyst (\%) & 71.4 & 57 \\
\hline 9. & Postoperative neurological deterioration (\%) & 71.4 & 28.6 \\
\hline 10. & No. of patients with McCormik grade $>\| 1$ at 5 years $(\%)$ & 14.3 & 14.3 \\
\hline 11. & Postoperative mortality & 0 & 1 \\
\hline 12. & Median duration of follow-up (mo) & 72 & 12 \\
\hline
\end{tabular}

VHL, von Hippel-Lindau; SI. No., serial number.

spinal tumors. Compared with patients with sporadic spinal hemangioblastomas, those with VHL disease had poor functional grades both preoperatively and in the immediate postoperative period $(p=0.038)$. At 1 - and 5 -year follow-up, there was no significant difference in functional outcomes between sporadic and VHLassociated spinal hemangioblastomas ( $p=0.10$ and 0.36 , respectively) (Table 2).

\section{Discussion}

Intramedullary spinal cord tumors are a rare and challenging pathological entity. Fedor Krause, in 1908, published the first surgical series on spinal tumors [4]. Since the introduction of microsurgery and modern imaging techniques, the results of surgery for intramedullary tumors have improved considerably. It is now widely accepted that surgery on these tumors should be recommended early before the onset of severe neurological deficits.

Hemangioblastomas are highly vascular tumors that represent approximately $3 \%$ of all tumors of CNS. They represent approximately $1.6 \%-14.8 \%$ of all intramedullary spinal tumors [5]. These tumors occur as sporadic lesions or as components of VHL syndrome, which accounts for $20 \%-30 \%$ of hemangioblastoma cases [6]. Although they are considered to be histologically benign, hemangioblastomas may cause significant neurological deficits, especially when they occur in the spinal cord.

The prevalence of VHL syndrome is between 1 in 35,000 and 1 in 40,000 [7]. The median life expectancy of a person with VHL syndrome is 49 years. The most common causes of death are neurological complications of hemangioblastomas (53\%) and metastatic clear cell RCC (32\%) [8]. Recent data shows RCC as the major cause of death [9]. This is because of advances in the management of CNS hemangioblastomas.

Diagnostic criteria for VHL include the following [10]: (1) More than one hemangioblastoma in CNS (brain, spinal cord) or eye, (2) A single hemangioblastoma in CNS or retina, plus a visceral manifestation (multiple renal, pancreatic, or hepatic cysts; pheochromocytoma; renal cancer), (3) Positive family history plus any one of the 
above clinical manifestations, (4) Elucidation of a deleterious mutation in the VHL gene.

Different VHL types and subtypes can be distinguished based on the predominant pathology in reference to pheochromocytomas, as described below [7,11-13]: (1) VHL type 1, renal carcinoma and hemangioblastoma; (2) VHL type 1B, VHL deletions that include the HSPC300 gene, characterized by protection from RCC; (3) VHL type 2A, hemangioblastoma and pheochromocytoma; (4) VHL type $2 \mathrm{~B}$, renal carcinoma and pheochromocytoma; (5) VHL type 2C, isolated pheochromocytoma.

\section{Incidence}

The proportion of spinal hemangioblastomas associated with VHL has been estimated be 13\%-59\% [14]. In a 10 -year clinical and genetic study of 213 patients with hemangioblastoma, Richard et al. [15] demonstrated that $34.3 \%$ of patients were affected with VHL. Compared with infratentorial lesions, spinal hemangioblastomas were found to be more frequently related to VHL. They are slightly more common in males, with male:female ratio being $1.6: 1$ to $5.5: 1$ [16]. In our series, the incidence was slightly higher in females (male:female ratio=1:1.3). These tumors occur in the third or fourth decade of life. Average age of onset has been estimated to be 33 years (in our series, it was 33.5 years).

\section{Location}

The most common locations for CNS hemangioblastomas are the cerebellum $(44 \%-72 \%)$, spinal cord $(13 \%-50 \%)$, brainstem $(10 \%-20 \%)$, supratentorial region $(<1 \%)$, and lumbosacral nerve roots $(<1 \%)$ [17]. Spinal hemangioblastomas are intramedullary tumors and most are found at the level of the cervical and dorsal vertebrae. They can occur throughout the spine from the cervical to the lumbosacral areas, affecting the spinal cord, nerve roots, conus medullaris, cauda equina, and filum terminale $[17,18]$. Most hemangioblastomas of spinal nerves arise from dorsal sensory fascicles [19]. Among the symptomproducing spinal hemangioblastomas, 95\% are associated with syringomyelia [20].

\section{Clinical presentation}

Symptoms are associated with the location of the tumor and the presence of edema, cyst, and/or syrinx. A syrinxcan result in a misleading clinical presentation because it can cause symptoms that localize to spinal cord segments remote from the tumor. Symptoms may range from mild to severe and include sensory or motor deficits, pain, dysesthesias, and bowel and urinary dysfunctions. Studies have shown that sensory dysfunction or pain is the most prevalent first clinical symptom. In our series, motor weakness was the predominant symptom ( $86 \%$ of patients). Sensory symptoms in the form of numbness and paresthesia were present in $79 \%$ of patients.

\section{Imaging}

A contrast-enhanced MRI is the diagnostic modality of choice for spinal hemangioblastomas. They are isointense on T1-weighted images and hyperintense on T2-weighted images, with homogeneous enhancement on contrast administration (Fig. 1) [18,21]. Symptomatic tumors are almost always associated with peritumoral edema and syrinx. Syrinx is assumed to arise from transudation of fluid from the tumor capillaries $[3,6,16,22]$. The incidence of a cyst component in the tumor has been reported to be $50 \%-70 \%$. In our series, tumor-associated syrinx was seen in $64 \%$ of cases. During removal of the solid part of the tumor, the syrinx is usually opened; after removing the solid part, the syrinx always stops growing and usually regresses in size [6].
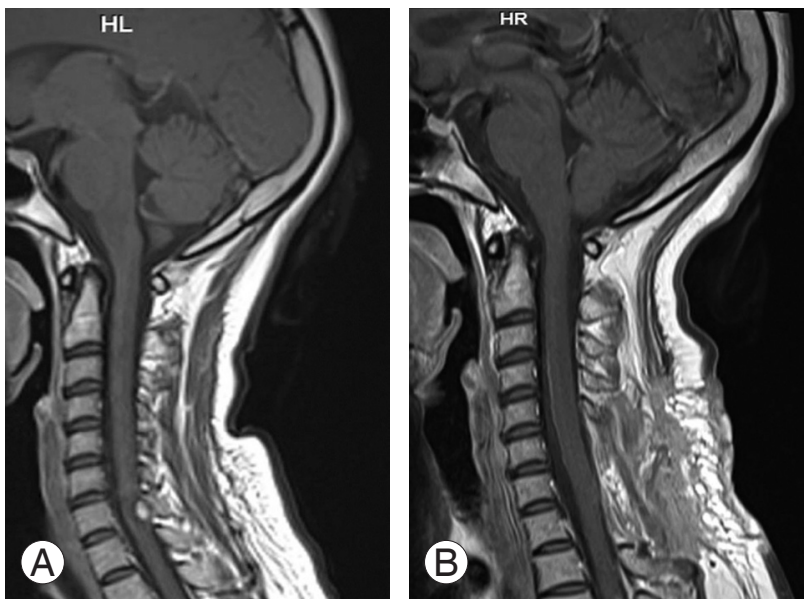

Fig. 1. (A) Preoperative T1-weighted contrast-enhanced midsagittal magnetic resonance imaging (MRI) of the cervical spine showing intensely enhancing hemangioblastoma at the level of C7 spine. (B) Postoperative midsagittal contrast-enhanced MRI of the same patient showing complete excision of the tumor. 


\section{Preoperative angiography and embolization}

Angiography can be useful during surgical planning to identify the feeding and draining vessels. Angiography was performed preoperatively in patients in whom the diagnosis was doubtful or whose MRIs showed multiple flow voids on T2-weighted images. Prominent tumor blush on angiogram and multiple flow voids are indirect indicators of high vascularity and almost diagnostic of a hemangioblastoma. In such patients, preoperative embolization can be attempted to reduce the intraoperative bleeding (Fig. 2) [23,24]. In our series, angiography was preoperatively performed in $79 \%$ of patients for treatment planning. Although embolization reduces the blood loss, it is not necessary for complete removal of the tumor because super-selective embolization of the feeding vessels may be difficult and might compromise the blood supply of the normal cord [25].

\section{Surgical management}

First successful removal of an intramedullary tumor was done by von Eiselsberg and Ranzi [26] in 1907. The surgical outcome was poor until the development of microneurosurgical techniques in the 1960s. The first successful removal of a spinal hemangioblastoma was performed by
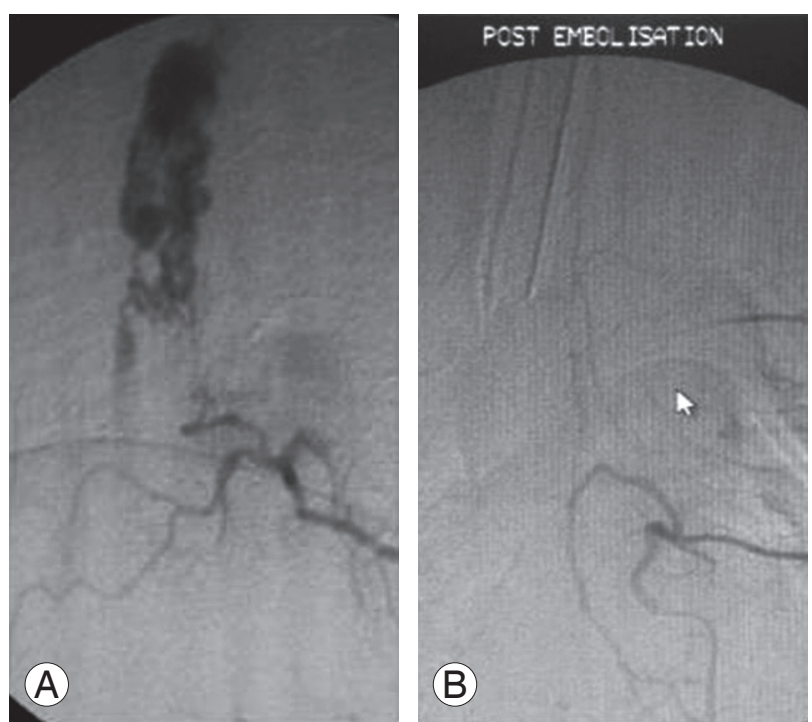

Fig. 2. (A) Digital subtraction angiography (DSA) of the dorsal spine showing tumor blush of a hemangioblastoma in the upper dorsal spine mimicking an arteriovenous malformation nidus. (B) DSA of the same patient after embolization showing complete obliteration of the tumor blush.
Dr. F. Schultze in 1912 [27].

Using the principles of microneurosurgery established by eminent neurosurgeons, we could achieve total excision in all patients. Major bleeding during the procedure should be avoided; this can be achieved by not entering the tumor. Manipulation of the spinal cord should be minimized to avoid damage to the nerve tissue and supplying vessels. Dissection in the plane between the tumor and spinal cord tissue with low-power coagulation is important for good postoperative outcomes.

\section{Surgical outcome}

Preoperative neurological function is correlated with postoperative neurological status [3]. In our series, patients who were functional grade I had good clinical outcomes. At 5-year follow-up, 78.6\% of patients had good functional outcomes. Most of the neurological deterioration seen in the immediate postoperative period was reversible. Of eight patients who had neurological deterioration in the postoperative period, six completely recovered at the end of 1-year follow-up. Tumor location and volume are the other predictors of poor outcome; patients with ventral or ventrolateral lesions have poor outcomes. All patients in our series had dorsally located tumors.

\section{Patients with VHL disease}

Patients with VHL disease often present with multiple asymptomatic hemangioblastomas. It is neither necessary nor possible to resect all the tumors. Therefore, most authors advocate operating on tumors that become symptomatic. However, some authors advise resecting asymptomatic tumors if the solid or pseudocystic part demonstrates progression, before possibly irreversible deficits occur [28]. Previous studies showed no difference in outcomes between sporadic and VHL-associated cases [16].

In our series, 7 (50\%) patients had VHL disease. This high rate may be attributable to the fact that our institution serves as a reference center. Among these seven patients, four had good functional outcomes and three had poor outcomes. Of the three patients with poor outcomes, two had poor functional grade preoperatively. Toward the end of the study period, there was a clustering of VHL-associated spinal hemangioblastoma patients, who could be followed up for a shorter duration only. 
Although patients with VHL-associated spinal tumors had a significantly poorer functional grade than those with sporadic hemangioblastomas in the preoperative and immediate postoperative period, at follow-up, there was no difference in the outcome between sporadic and VHL-associated cases.

\section{Recurrence}

Recurrence may be due to incomplete removal of the tumor or growth of new tumors in both sporadic and VHL-associated cases. In a study of 13 patients, comprising 6 patients with VHL-associated and 7 with sporadic hemangioblastomas, Miyagami and Katayama [29] noted recurrence in sites remote from the primary region in all patients with VHL-associated tumor. In contrast, no patients with sporadic tumors had recurrence over a period of 5-22 years.

In a study of the long-term prognosis of CNS hemangioblastomas, Niemela et al. [30] reported recurrence in both VHL-associated and sporadic hemangioblastomas, despite complete surgical removal. They reported a median time to recurrence of 19 years in patients with VHL syndrome and 11 years in sporadic disease. Clinical features reported to correlate with recurrence include young age at initial diagnosis, evolution of related symptoms over 6-12 months, multicentricity of CNS tumors, and the presence of VHL syndrome [30].

In our series, there was recurrence in $1(7.1 \%)$ patient at 3-year follow-up, despite complete removal. This patient (a female) had recurrence at the operated site and also at a distant site. She had all the risk factors mentioned above, including being young at presentation (20 years), having short duration of symptoms (6 months), and having VHL disease.

The documented postoperative complications are CSF leaks, wound infections, aseptic meningitis, and spinal deformity following multilevel laminectomies. In our series, 1 (7.1\%) had postoperative CSF leak, which was managed by wound re-suturing; none had wound infection. Most of our patients required a two- or three-level laminectomy. None of them developed any spinal deformity or required an additional fusion. Extensive laminectomies are not necessary to expose the polar cysts. Limiting the laminectomy to expose only the tumor usually prevents additional fusion.

\section{Conclusions}

Microsurgical excision of spinal hemangioblastomas can cause postoperative morbidity, mainly in the form of neurological deterioration. Almost half of our patients had deterioration in McCormick grade in the immediate postoperative period. However, complete microsurgical excision can result in good long-term functional outcomes, as most of the immediate postoperative neurological deterioration was reversible. There was no difference in the long-term functional outcomes between sporadic and VHL-associated spinal hemangioblastomas. It is important to plan surgical excision before a significant morbidity develops in a patient with spinal hemangioblastoma.

\section{Conflict of Interest}

No potential conflict of interest relevant to this article was reported.

\section{ORCID}

Joe M. Das: https://orcid.org/0000-0002-6373-1500

\section{References}

1. Butman JA, Linehan WM, Lonser RR. Neurologic manifestations of von Hippel-Lindau disease. JAMA 2008;300:1334-42.

2. Catapano D, Muscarella LA, Guarnieri V, Zelante L, D'Angelo VA, D'Agruma L. Hemangioblastomas of central nervous system: molecular genetic analysis and clinical management. Neurosurgery 2005;56: 1215-21.

3. Lonser RR, Weil RJ, Wanebo JE, DeVroom HL, Oldfield EH. Surgical management of spinal cord hemangioblastomas in patients with von Hippel-Lindau disease. J Neurosurg 2003;98:106-16.

4. Klekamp J. Treatment of intramedullary tumors: analysis of surgical morbidity and long-term results. J Neurosurg Spine 2013;19:12-26.

5. Browne TR, Adams RD, Roberson GH. Hemangioblastoma of the spinal cord: review and report of five cases. Arch Neurol 1976;33:435-41.

6. Neumann HP, Eggert HR, Weigel K, Friedburg H, Wiestler OD, Schollmeyer P. Hemangioblastomas of the central nervous system: a 10-year study with 
special reference to von Hippel-Lindau syndrome. J Neurosurg 1989;70:24-30.

7. Neumann HP, Wiestler OD. Clustering of features of von Hippel-Lindau syndrome: evidence for a complex genetic locus. Lancet 1991;337:1052-4.

8. Neumann HP, Eggert HR, Scheremet R, et al. Central nervous system lesions in von Hippel-Lindau syndrome. J Neurol Neurosurg Psychiatry 1992;55:898901.

9. Leung RS, Biswas SV, Duncan M, Rankin S. Imaging features of von Hippel-Lindau disease. Radiographics 2008;28:65-79.

10. Gupte S. Recent advances in pediatrics, special volume, 18: pediatric neurology. New Delhi: Jaypee Bros. Medical Publishers; 2008.

11. Brauch H, Kishida T, Glavac D, et al. Von HippelLindau (VHL) disease with pheochromocytoma in the Black Forest region of Germany: evidence for a founder effect. Hum Genet 1995;95:551-6.

12. Hoffman MA, Ohh M, Yang H, Klco JM, Ivan M, Kaelin WG Jr. von Hippel-Lindau protein mutants linked to type $2 \mathrm{C}$ VHL disease preserve the ability to downregulate HIF. Hum Mol Genet 2001;10:1019-27.

13. McNeill A, Rattenberry E, Barber R, Killick P, MacDonald F, Maher ER. Genotype-phenotype correlations in VHL exon deletions. Am J Med Genet A 2009;149:2147-51.

14. Na JH, Kim HS, Eoh W, Kim JH, Kim JS, Kim ES. Spinal cord hemangioblastoma: diagnosis and clinical outcome after surgical treatment. J Korean Neurosurg Soc 2007;42:436-40.

15. Richard S, David P, Marsot-Dupuch K, Giraud S, Beroud C, Resche F. Central nervous system hemangioblastomas, endolymphatic sac tumors, and von Hippel-Lindau disease. Neurosurg Rev 2000;23:1-22.

16. Conway JE, Chou D, Clatterbuck RE, Brem H, Long DM, Rigamonti D. Hemangioblastomas of the central nervous system in von Hippel-Lindau syndrome and sporadic disease. Neurosurgery 2001;48:55-62.

17. Pluta RM, Wait SD, Butman JA, et al. Sacral hemangioblastoma in a patient with von Hippel-Lindau disease: case report and review of the literature. Neurosurg Focus 2003;15:E11.

18. Choyke PL, Glenn GM, Walther MM, Patronas NJ, Linehan WM, Zbar B. von Hippel-Lindau disease: genetic, clinical, and imaging features. Radiology
1995;194:629-42.

19. Glasker S, Berlis A, Pagenstecher A, Vougioukas VI, Van Velthoven V. Characterization of hemangioblastomas of spinal nerves. Neurosurgery 2005;56:503-9.

20. Wanebo JE, Lonser RR, Glenn GM, Oldfield EH. The natural history of hemangioblastomas of the central nervous system in patients with von Hippel-Lindau disease. J Neurosurg 2003;98:82-94.

21. Xu QW, Bao WM, Mao RL, Yang GY. Magnetic resonance imaging and microsurgical treatment of intramedullary hemangioblastoma of the spinal cord. Neurosurgery 1994;35:671-5.

22. Malis LI. Atraumatic bloodless removal of intramedullary hemangioblastomas of the spinal cord. J Neurosurg 2002;97:1-6.

23. Eskridge JM, McAuliffe W, Harris B, Kim DK, Scott J, Winn HR. Preoperative endovascular embolization of craniospinal hemangioblastomas. AJNR Am J Neuroradiol 1996;17:525-31.

24. Tampieri D, Leblanc R, TerBrugge K. Preoperative embolization of brain and spinal hemangioblastomas. Neurosurgery 1993;33:502-5.

25. Roonprapunt C, Silvera VM, Setton A, Freed D, Epstein FJ, Jallo GI. Surgical management of isolated hemangioblastomas of the spinal cord. Neurosurgery 2001;49:321-7.

26. von Eiselsberg AF, Ranzi E. Ueber die chirurgische behandlung der hirn-und ruckenmarkstumoren. Arch Klin Chir 1913;102:309-468.

27. Cushing H, Bailey P. Tumours arising from the blood vessels of the brain: angiomatous malformations and hemangioblastomas. Springfield: Charles C Thomas; 1928.

28. Van Velthoven V, Reinacher PC, Klisch J, Neumann $\mathrm{HPH}$, Glasker S. Treatment of intramedullary hemangioblastomas, with special attention to von hippel-lindau disease. Neurosurgery 2003;53:1306-14.

29. Miyagami M, Katayama Y. Long-term prognosis of hemangioblastomas of the central nervous system: clinical and immunohistochemical study in relation to recurrence. Brain Tumor Pathol 2004;21:75-82.

30. Niemela M, Lemeta S, Summanen P, et al. Long-term prognosis of haemangioblastoma of the CNS: impact of von Hippel-Lindau disease. Acta Neurochir (Wien) 1999;141:1147-56. 